sich bei Gewässern um sehr dynamische Lebensräume handelt, sodass sich zumindest unter ökologischen Gesichtspunkten vergleichsweise schnell neue verfestigte Zustände einstellen, werden solche deutlich kürzeren Zeiträume sogar eher die Regel als die Ausnahme sein. Dies belegen auch die Erfahrungen im Zusammenhang mit der naturschutzrechtlichen Eingriffsregelung, wo sich gezeigt hat, dass in den meisten Fällen ab einem Zeitraum von bereits zehn Jahren Ausgleichs- und Ersatzmaßnahmen keiner Entwicklungspflege mehr bedürfen, weil sich nach diesem Zeitraum die Verhältnisse ausreichend verfestigt haben. ${ }^{52}$ Liegen daher für die Gestalt des Gewässers maßgebliche Eingriffe oder Ereignisse zehn Jahre oder mehr zurück, spricht der erste Anschein für den Eintritt neuer verfestigter Verhältnisse. Dieser neue Zustand kann dann nicht einfach durch entsprechende behördliche Anordnung rückgängig gemacht werden. Vielmehr würde sich dies seinerseits als Gewässerausbau darstellen, der gemäß \68 Abs. 1 WHG grundsätzlich der Planfeststellung bedarf.

Bezogen auf die nachträgliche Legalisierung einer formell illegalen Anlage ist jedoch darauf hinzuweisen, dass die Frage der verfestigten Verhältnisse lediglich für das Vorliegen eines Gewässerausbaus relevant ist. Die Rechtsfolge dieses Befunds besteht indes lediglich darin, dass bejahendenfalls gemäß $\$ 68$ Abs. 1 und 2 WHG ein Planfeststellungs- oder Plangenehmigungsverfahren durchzuführen ist. Von welchem Zustand bei Prüfung der materiell-rechtlichen Voraussetzungen für die Zulassung des Vorhabens im Planfeststellung- bzw. Plangenehmigungsverfahren auszugehen ist, bestimmen indes weder $\$ 67$ Abs. 2 Satz 1 noch $\$ 68$ WHG, sondern beurteilt sich ausschließlich nach dem einschlägigen materiellen Recht (dazu bereits oben 3.1 bis 3.5).

\section{Fazit}

Demnach kann festgehalten werden, dass der Grundsatz der Maßgeblichkeit der Sach- und Rechtslage zum Zeitpunkt der Genehmigungserteilung auch im Fall der nachträglichen Legalisierung gilt. Will die Verwaltung verhindern, dass der Antragsteller in den Genuss der Früchte illegalen Handelns kommt, muss sie entsprechend den dafür bereitgehaltenen gesetzlichen Ermächtigungsgrundlagen und regelmäßig in Ausübung pflichtgemäßen Ermessens repressiv gegen jenes Handeln bzw. dessen Resultate vorgehen. Diese Korrekturmaßnahmen in Gestalt von Nutzungsuntersagungen, Wiederherstellungsanordnungen sowie Rückbau- oder Kompensationsverfügungen sind dann auch in dem der nachträglichen Legalisierung dienenden Genehmigungsverfahren zu berücksichtigen. Um Zirkelschlüsse zu vermeiden, kann im Rahmen der Ermessensausübung zum Erlass der jeweiligen Korrekturmaßnahme die Legalisierungsfähigkeit des rechtswidrigen Handelns lediglich in den Fällen ermessensleitend sein, in denen durch die rechtswidrige Schaffung von Fakten keine genehmigungsrelevanten Umstände beeinflusst worden sind. Beim Gewässerausbau sind einem repressiven Einschreiten überdies dahingehend Grenzen gesetzt, dass hier neue verfestigte Verhältnisse selbst dann den Maßstab bilden, wenn sie illegal geschaffen wurden. In diesem Fall bedarf die Wiederherstellung des Ausgangszustands ihrerseits der Planfeststellung. Solange ein entsprechender Planfeststellungsbeschluss nicht vorliegt, ist der tatsächlich vorfindliche Zustand maßgeblich.

52) Fischer/Zeidler, NuL 2009, 209, 210.

\title{
Beiräte als Instrument einer Ernährungswende - Die Etablierung von Ernährungsräten in Deutschland
}

\author{
Annelie Sieveking und Thomas Schomerus
}

\begin{abstract}
C Der/die Autor(en) 2020. Dieser Artikel ist eine Open-Access-Publikation.
Deutschlandweit sind Ernährungsräte im Kommen. Diese im angelsächsischen Raum als ,food policy councils" bekannten Gremien sind überwiegend zivilgesellschaftlich initiiert und versuchen z.B. durch Öffentlichkeitsarbeit oder Forderungen an Politik und Verwaltung eine Ernährungswende voranzubringen. Der Beitrag untersucht die Möglichkeiten einer stärkeren rechtlichen Verankerung dieser Gremien.
\end{abstract}

\section{Einleitung}

Ernährung ist ein wichtiger Teilaspekt von Nachhaltigkeit. Ziel 2 der 17 globalen Ziele für nachhaltige Entwicklung lautet: „Den Hunger beenden, Ernährungssicher-

Annelie Sieveking MSc, Wissenschaftliche Mitarbeiterin und Doktorandin am Institut für Nachhaltigkeitssteuerung, Leuphana Universität,

Lüneburg, Deutschland

Prof. Dr. Thomas Schomerus, RiOVG, Inhaber des Lehrstuhls für Öffentliches Recht, insbes. Energie- und Umweltrecht, Leuphana Universität,

Lüneburg, Deutschland heit und eine bessere Ernährung erreichen und eine nachhaltige Landwirtschaft fördern". ${ }^{1}$ Auch wenn Hunger im engeren Sinne in Deutschland und der EU kein vordringliches Problem darstellt, ist doch die Qualität der Ernährung immer stärker in den Fokus der interessierten Öffentlichkeit gerückt. Gesunde und nachhaltige Ernährung stellt auch hier eine große Herausforderung dar. Sie ist ein wichtiges Thema im Hinblick auf Gesundheit, Umwelt und Soziales. Ernährungsbedingte Krankheiten scheinen zuzunehmen. ${ }^{2}$ Unter dem Umweltaspekt wird die Verschwendung von Ressourcen immer mehr thematisiert. Studien zeigen auf, dass pro Jahr und Kopf in Deutschland ca. $82 \mathrm{~kg}$ Lebensmittel in den privaten Haushalten zu

1) S. Bundesministerium für wirtschaftliche Zusammenarbeit und Entwicklung, Die globalen Ziele für nachhaltige Entwicklung, Ziel 2, Stand 1.9.2019 abrufbar unter https://www.bmz.de/de/ministerium/ziele/2030_agenda/17_ziele/ziel_002_hunger/index.html.

2) S. etwa: Ungesunde Ernährung ist weltweit größtes Krankheitsrisiko, 2015, Stand 16.8.2020, abrufbar unter https://healthcarein-europe.com/de/news/ungesunde-ernaehrung-ist-weltweitgroesstes-krankheitsrisiko.html. 
Abfall werden. ${ }^{3}$ Die Vermeidung von Lebensmittelabfällen war ein wesentlicher Beweggrund für die Änderung der Abfallrahmenrichtlinie, ${ }^{4}$ was wiederum in der beabsichtigten Novelle des KrWG im Abfallvermeidungsprogramm in $\$ 33$ Abs. 3 Nr. 2 g aufgenommen wurde. ${ }^{5}$ Hinzu kommt die erhebliche Klimarelevanz unserer Ernährung. Auf den verschiedenen Wertschöpfungsstufen für Lebensmittel und durch die erforderlichen Transporte entstehen erhebliche Treibhausgasemissionen, die etwa 23 Prozent der jährlichen Gesamtemissionen Deutschlands ausmachen. ${ }^{6}$ Bestimmte Ernährungsformen schlagen hier besonders zu Buche. So ist das Treibhauspotenzial tierischer etwa viermal so hoch wie das pflanzlicher Produkte. ${ }^{7}$

Mit diesen und vielen weiteren Aspekten des Gesamtbereichs von Ernährung und Landwirtschaft befassen sich Ernährungsräte als neue Akteure neben den bisherigen etablierten Interessenvertretern aus der Agrarwirtschaft, der lebensmittelverarbeitenden Industrie, den Verbraucher- und Umweltverbänden und nicht zuletzt den staatlichen und kommunalen Institutionen. Ernährungsräte können als ein Unterfall der auf ein breiteres Themenspektrum gerichteten Nachhaltigkeitsräte angesehen werden. Nachhaltigkeitsräte wurden als Gremien bezeichnet, ,die von Organisationen einberufen werden, um diese im Hinblick auf deren nachhaltiges Handeln zu beraten" ${ }^{8}$ Übertragen auf Ernährungsräte können diese definiert werden als Gremien, deren wesentliche Aufgabe darin liegt, relevante Akteure in der gesamten Lebensmittelwertschöpfungskette von Produktion, Verarbeitung, Transport, Konsum und Entsorgung einschließlich der Vermeidung von Lebensmittelabfällen im Hinblick auf Nachhaltigkeit $\mathrm{zu}$ informieren und zu beraten. Sie können auch nur einzelne dieser in vielen ernährungsrelevanten Themen adressieren. Ein Ernährungsrat kann in diesem Sinne verstanden werden als , ,...] eine Multi-Akteurs-Plattform, in der sich nach Möglichkeit alle diejenigen, die relevantes Wissen über, Einflussmöglichkeiten auf, Interesse an oder Ressourcen für ein nachhaltiges lokales Ernährungssystem haben, versammeln“. ${ }^{9}$ Die Zusammensetzung der Ernährungsräte im deutschsprachigen Raum variiert, dennoch sind in den meisten Fällen Personen aus Zivilgesellschaft, Lebensmittelwirtschaft sowie Politik und Verwaltung involviert.

Potenzieller Adressat der Ernährungsräte ist neben der Politik vor allem auch die Öffentlichkeit. Sie können in Projekten, in Arbeitsgruppen oder in alternativen Formen auf das derzeitig vorherrschende Ernährungssystem einwirken und insbesondere dazu beitragen, den Kontakt zwischen Erzeugern und Verbrauchern sowie anderen Akteuren (wieder)herzustellen. Sie können das Bewusstsein in der Bevölkerung für eine nachhaltige Ernährung stärken, aber auch politische Entscheidungen in diese Richtung beeinflussen. Auf diese Weise kann die Etablierung von Ernährungsräten einen Hebelpunkt für die nachhaltige Entwicklung ${ }^{10}$ darstellen. ${ }^{11}$

Der Beitrag befasst sich mit der Frage, wie und auf welchen Ebenen Ernährungsräte in Deutschland bereits aktiv sind. Darüber hinaus wird untersucht, welche rechtlichen Rahmenbedingungen möglich und geeignet sind, um Ernährungsräte als neue Akteure einer Ernährungswende zu stärken. Letztlich geht es darum, welche Chancen und Möglichkeiten Ernährungsräte bieten, um eine Ernährungswende voranzubringen.

Dazu wird im Folgenden zunächst kurz geschildert, wo und in welcher Form sich bereits Ernährungsräte etabliert haben (2.). Darauf werden Einblicke in Ernährungsräte als Akteure einer Ernährungswende gegeben und es wird der Frage nachgegangen, wie insbesondere Politik und Verwaltung durch diese beraten werden können (3.). Unter 4. geht es dann um den derzeitigen rechtlichen Status von Ernährungsräten in Deutschland und die Möglichkeiten einer stärkeren rechtlichen Verankerung von Ernährungsräten, um schließlich ein Fazit zu ziehen (5.).

\section{Die Etablierung von Ernährungsräten}

In den Vereinigten Staaten und Kanada existieren Ernährungsräte, sogenannte ,food policy councils", schon seit mehreren Jahrzehnten. Die ersten Räte entstanden Anfang der 1980er Jahre in den USA, bzw. Anfang der 1990er Jahre in Kanada. Der aktuelle Bericht des Johns Hopkins Center for a Livable Future verdeutlicht, dass die Anzahl von Ernährungsräten in den USA und Kanada seitdem kontinuierlich zugenommen hat, mit einem besonderen Anstieg in den letzten zehn Jahren. Derzeit wird von 341 aktiven Ernährungsräten auf verschiedenen politischen Ebenen berichtet, wobei die Mehrheit (71 Prozent) auf lokaler Ebene agiert. ${ }^{12}$ Die Ernährungsräte der ersten Stunde entstanden häufig aus informellen Koalitionen in den Bereichen Hungerbekämpfung, nachhaltige Landwirtschaft und Kommunalentwicklung. ${ }^{13}$ Begrenzter Zugang zu gesunden Lebensmitteln und ein Mangel an Koordination in Bezug auf Ernährungsplanung führten 1982 zur Gründung des ersten Ernährungsrats in der Stadt Knoxville, Tennessee, der seitdem viele Aktivitäten zur Verbesserung der Situation (z.B. im Bereich Schulverpflegung) angestoßen hat. ${ }^{14}$ Während anfangs ein Schwerpunkt auf sozialen Themen lag, verfolgen Ernährungsräte heute einen breiteren Ansatz, der soziale, umwelt-und gesundheitsbezogene sowie wirtschaftliche Aspekte miteinbezieht und Menschen zusammenbringt, die sich in verschiedenen Bereichen für das Thema Ernährung engagieren. ${ }^{15}$

In Europa gründeten sich erst in der vergangenen Dekade die ersten Ernährungsräte, überwiegend in Großbritannien (z. B. Bristol und Cork). Seit Kurzem gibt es auch in anderen europäischen Ländern erste Bestrebungen, ver-

3) Kranert et al., 2012, Ermittlung der weggeworfenen Lebensmittelmengen und Vorschläge zur Verminderung der Wegwerfrate bei Lebensmitteln in Deutschland.

4) S. Art. 9 Abs. 1 lit. g) der Änderungsrichtlinie (EU) 2018/851 vom 30.5.2018 zur Anderung der Richtlinie 2008/98/EG über Abfälle, ABl. 2018 L 150, S. 109.

5) Gesetzentwurf der Bundesregierung, Entwurf eines Gesetzes zur Umsetzung der Abfallrahmenrichtlinie der Europäischen Union, Bearbeitungsstand: 3.2.2020, abrufbar unter https://www. $\mathrm{bmu}$.de/gesetz/referentenentwurf-eines-gesetzes-zur-umsetzung-der-abfallrahmenrichtlinie-der-europaeischen-union/.

6) Jepsen/Vollmer/Eberle/Fels/Schomerus, Entwicklung von Instrumenten zur Vermeidung von Lebensmittelabfällen, TEXTE 85/2016 des Umweltbundesamts, S. $22 \mathrm{f}$.

7) Ebenda, S. $24 \mathrm{f}$.

8) Schomerus, Nachhaltigkeit braucht Institutionen - zur Institutionalisierung von Nachhaltigkeitsräten, NuR 2011, 1, 2.

9) Wissmann, in: Antoni-Komar/Kropp/Paech/Pfriem (Hrsg.), Transformative Unternehmen und die Wende in der Ernährungswirtschaft, 2019, S. 317.

10) Vgl. Abson/Abernethy/Fischer/Lang/Leventon/Ives/Newig/Schomerus/ Vilsmaier/von Wehrden, Leverage points for sustainability transformation: Institutions, people and knowledge, 2016, Ambio 46(1):30.

11) Das Potenzial von Ernährungsräten als Hebelpunkte für nachhaltige Entwicklung wird in der Doktorarbeit von Annelie Sieveking, die sich derzeit noch im Verfahren befindet, ausführlich untersucht (Sieveking (unv.): Food Policy Councils: Levers for Sustainability Transformnation?)

12) Bassarab/Santo/Palmer, 2019, Food Policy Council Report 2018, Stand 1.7.2019, abrufbar unter http://www.foodpolicynetworks. org/food-policy-resources/, S. 3.

13) Pothukuchi/Kaufman, 1999, Placing the food system on the urban agenda: The role of municipal institutions in food systems planning, Agriculture and Human Values, 16, S. 219.

14) Harper/Shattuck/Holt-Giménez/Wolf/Workman/Clare-Roth/ElKhoury/Turrell/Strong, 2009, Food Policy Councils: Lessons Learned. Food First Institute for Food and Policy, Stand 1.7.2019, abrufbar unter https://foodfirst.org/publication/food-policycouncils-lessons-learned/, S. 17.

15) Stierand, Speiseräume. Die Ernährungswende in der Stadt, 2014, S. 169. 
gleichbare Gremien zu etablieren, so z.B. in den Niederlanden oder in Italien ${ }^{16}$. In Deutschland wurden 2016 die ersten beiden Ernährungsräte in den Großstädten Köln und Berlin gegründet. Seitdem gab es eine sehr dynamische Entwicklung im deutschsprachigem Raum. Bei einem ersten Treffen der Initiativen im Herbst 2017 kamen über 100 Personen aus über 40 verschiedenen Orten zusammen, um sich zu vernetzen und Erfahrungen bei der Gründung von Ernährungsräten auszutauschen. Rund ein Dutzend weitere Räte haben sich mittlerweile formiert, überwiegend in Städten, aber auch vereinzelt auf anderen politischadministrativen Ebenen (z.B. 2018 im Landkreis Fürstenfeldbruck oder $2020 \mathrm{im}$ Land Brandenburg ${ }^{17}$ ). Außerdem gibt es derzeit an über vierzig Orten im deutschsprachigem Raum Initiativen, die die Gründung von weiteren Ernährungsräten vorbereiten. ${ }^{18}$ Beim zweiten Netzwerktreffen der Gründungsinitiativen und der bestehenden Ernährungsräte im Herbst 2018 kamen mehr als 150 Vertreterinnen und Vertreter aus Deutschland, Österreich, der Schweiz, Luxemburg und den Niederlanden zusammen und verabschiedeten als Leitlinie für die Arbeit eine erste gemeinsame Erklärung mit dem Titel „Ernährungsdemokratie jetzt". ${ }^{19}$

\section{Ernährungsräte als Akteure einer Ernährungswende in Deutschland}

\subsection{Begleitung der Etablierung von Ernährungsräten durch Politik und Verwaltung}

Bisher gab es vereinzelte Initiativen seitens der Politik, die Gründung von Ernährungsräten anzustoßen. In Bielefeld stellte die CDU-Fraktion im Oktober 2016 einen Antrag im Ausschuss für Klima und Umwelt, die Einführung eines Ernährungsrates für Bielefeld durch die Verwaltung zu prüfen und wenn möglich umzusetzen. ${ }^{20}$ Auf diesen einstimmig angenommenen Antrag folgte eine erste Auftaktveranstaltung mit verschiedenen Vertreterinnen und Vertretern von Organisationen, Vereinen und Institutionen, die sich mit Ernährung befassen sowie die Installation einer Kerngruppe, welche die weiteren Treffen koordinierte. Bei der Gründung des Bielefelder Ernährungsrates im November 2018 kamen ca. 75 engagierte Menschen aus unterschiedlichen Bereichen zusammen, unterzeichneten symbolisch die im Vorfeld erarbeitete „Bielefelder Charta für gutes und gesundes Essen" und gründeten drei Arbeitsgruppen. ${ }^{21}$ Der Bielefelder Ernährungsrat ist kein städtisches Gremium, aber der gesamte Prozess wurde und wird von der Verwaltung begleitet. ${ }^{22}$

Im Landkreis Fürstenfeldbruck wurde die Gründung des ersten deutschen Ernährungsrates auf Landkreisebene auf einer Regionalkonferenz angestoßen, die vom Regionalmanagement veranstaltet wurde. Die Initiierung eines Ernährungsrates wurde als das meist favorisierte Projekt vorgeschlagen und im Folgenden vom Agenda-21-Büro des Landkreises vorangebracht. ${ }^{23} \mathrm{Nach}$ der Einladung zu einer ersten Veranstaltung zur Vernetzung interessierter Akteurinnen und Akteure wurden ein Gründungsausschuss und später vier thematische Arbeitsgruppen gegründet, die Projekte in verschiedenen Bereichen verfolgen. Das Engagement der Beteiligten wird weiterhin vom Agenda 21-Büro organisatorisch unterstützt, z.B. durch die Einladung zu Treffen oder das Verfassen von Protokollen. ${ }^{24}$

Die dargestellten Beispiele verdeutlichen, dass in Deutschland bereits vereinzelt die Gründung von Ernährungsräten von Politik und Verwaltung angestoßen wurde. Dennoch sind die bisher in Deutschland gegründeten Ernährungsräte zur großen Mehrheit auf zivilgesellschaftliche Initiative hin entstanden. Auch wenn in vielen Fällen Mitglieder aus Politik und Verwaltung involviert sind, berichteten bei den Netzwerktreffen der Initiativen auch viele davon, dass es schwierig war, mit Politik und Verwaltung ins Gespräch zu kommen. ${ }^{25}$
Andere Initiativen möchten zwar mit Personen aus Politik und Verwaltung zusammenarbeiten, aber diese nicht als Mitglieder in das Gremium involvieren, wie z.B. der Ernährungsrat Berlin, der sich als breites zivilgesellschaftliches Bündnis im Sinne einer ,unabhängigen Interessensvertretung ernährungspolitisch engagierter Bürger*innen der Stadt ${ }^{\text {'26 }}$ versteht.

\subsection{Beratung von Politik und Verwaltung durch Ernährungsräte}

Auch wenn auf Landesebene bisher seitens der Politik noch keine Gründung von Ernährungsräten angestoßen wurde, haben dennoch mehrere Bundesländer in den letzten Jahren Beratungsbedarf im Kontext einer Ernährungswende artikuliert. In einem speziellen Themenbereich, der Vermeidung von Lebensmittelabfällen, wurden Runde Tische zum Thema Lebensmittelwertschätzung einberufen. In NRW z.B. lädt das Verbraucherschutzministerium seit 2010 jährlich verschiedene Vertreterinnen und Vertreter aus Landwirtschaft, Einzelhandel, Lebensmittelwirtschaft, Wissenschaft und Verbraucherschutz ein, um ,[...] die Aufmerksamkeit und die Sensibilität für das Thema Lebensmittelverschwendung entlang der gesamten Lebensmittelwertschöpfungskette $z u$ erhöhen und das Bewusstsein für den Wert der Lebensmittel zu steigern" ${ }^{\prime 27}$. Durch den Austausch wurden landesweit verschiedene Projekte, wie z.B. die Nachhaltigen Ernährungstage NRW, initiiert. ${ }^{28}$ Auch in anderen Bundesländern wurden in den letzten Jahren vergleichbare Runde Tische eingesetzt, z. B. in Hamburg (2015), in Niedersachsen (2016) oder im Saarland (2016). Die Bundesländer mit ihren bereits bestehenden Aktivitäten wie z. B. der Förderung einer Vernetzung der Akteurinnen und Akteuren in Runden

16) Forno/Maurano, 2016, CIBO, sostenibilità e territorio. dai sistemi di approvvigionamento alternativi ai food policy councils. $R i$ vista Geografica Italiana, 123(1).

17) Der Ernährungsrat Brandenburg versteht sich als Zusammenschluss der regionalen Ernährungsratsinitiativen in Brandenburg und wurde am 13.12020 in Potsdam gegründet. Weitere Informationen, Stand 16.8.2020, abrufbar unter https://ernaehrungsrat-brandenburg.de/aktuelles/.

18) Eine Übersicht über die Gründungsinitiativen im deutschsprachigen Raum ist zu finden in Thurn/Oertel/Pohl, Genial lokal. So kommt die Ernährungswende in Bewegung, 2018, S. 192-197 und auf der Homepage des Netzwerks der Ernährungsräte, Stand 16.8.2020, abrufbar unter http://ernaehrungsraete.org/.

19) Netzwerk der Ernährungsräte, 2019, Ernährungsräte verabschieden Frankfurter Erklärung für gute Ernährung und Produktion, Stand 1.7.2019, abrufbar unter http://ernaehrungsraete.org/2018/11/27/frankfurter_erklaerung/.

20) CDU-Fraktion des Rates der Stadt Bielefeld, Antrag zur Sitzung des Ausschusses für Umwelt und Klima am 4.10.2016 (Drs. 3732/2014-2020).

21) Umweltamt Bielefeld, Beschlussvorlage der Verwaltung zum Ernährungsrate Bielefeld zur Sitzung des Ausschusses für Umwelt und Klimaschutz am 15.1.2019 (Drs. 7795/2014-2020).

22) Gespräch mit Frau Kleiner vom Dezernat Umwelt/Klimaschutz der Stadt Bielefeld am 3. 7.2019.

23) Landkreis Fürstenfeldbruck, Ernährungsrat für den Landkreis Fürstenfeldbruck, Stand 5.7.2019, abrufbar unter https://www.ernaehrungsrat-ffb.de/.

24) Gespräch mit Frau Bock vom Agenda 21-Büro des Landkreises Fürstenfeldbruck am 5.7.2019.

25) Gespräche mit Vertreterinnen und Vertreter verschiedener Ernährungsratsinitiativen bei den bisherigen Kongressen des Netzwerks der Ernährungsräte in Essen (2017) und Frankfurt (2018).

26) Ernährungsrat Berlin, 2017, Ernährungsdemokratie für Berlin, Stand 5.7.2019, abrufbar unter http://ernaehrungsrat-berlin.de/ ernaehrungsdemokratie-fuer-berlin/.

27) Ministerium für Umwelt, Landwirtschaft, Natur- und Verbraucherschutz des Landes Nordrhein-Westfalen, Lebensmittelverluste reduzieren - Wertschätzung für Lebensmittel erhöhen, Stand 4.7.2019, abrufbar unter https://www.umwelt.nrw.de/ verbraucherschutz/konsum-und-wertschaetzung-von-lebensmitteln/.

28) S. ebenda. 
Tab. 1 Rechtliche Rahmenbedingungen für Ernährungsräte als pluralistisch besetzte, langfristig eingerichtete Beratungsgremien

\begin{tabular}{|c|c|c|}
\hline & De lege lata & De lege ferenda \\
\hline Bundesebene & $\begin{array}{l}\text { Langfristige Beratungsgremien: nur ausgewählte Mitglieder, } \\
\text { keine Einbeziehung Externer }\end{array}$ & $\begin{array}{l}\text { Schaffung einer Rechtsgrundlage (Inhalte: Gründung } \\
\text { von Ernährungsräten, Aufgaben, Mitglieder)? }\end{array}$ \\
\hline Länderebene & $\begin{array}{l}\text { Langfristige Beratungsgremien: nur ausgewählte Mitglieder, } \\
\text { keine Einbeziehung Externer }\end{array}$ & $\begin{array}{l}\text { Schaffung einer Rechtsgrundlage (Inhalte: Gründung } \\
\text { von Ernährungsräten, Aufgaben, Mitglieder)? } \\
\text { Ausdehnung bestehender Runder Tische zur Wertschät- } \\
\text { zung von Lebensmitteln? }\end{array}$ \\
\hline $\begin{array}{l}\text { Kommunale } \\
\text { Ebene }\end{array}$ & $\begin{array}{l}\text { Rechtliche Möglichkeit zur Einrichtung von Gremien, z.B. \71 } \\
\text { NKomVG: Ausschüsse der Vertretung } \\
\text { Unterscheidung zwischen Beratungs- und Entscheidungsgremien } \\
\text { Obligatorische Einrichtung bestimmter Gremien }\end{array}$ & $\begin{array}{l}\text { Ernährungsräte als obligatorische Gremien im Kommunal- } \\
\text { verfassungsrecht? }\end{array}$ \\
\hline
\end{tabular}

Tischen werden als wichtige Partner bei der Umsetzung der Nationalen Strategie zur Reduzierung der Lebensmittelverschwendung benannt. ${ }^{29}$ Ziel dieser Strategie, die im Februar 2019 vom Bundeskabinett verabschiedet wurde, ist es, im Einklang mit der Agenda 2030 für Nachhaltige Entwicklung der Vereinten Nationen die Verschwendung von Lebensmitteln bis zum Jahr $2030 \mathrm{zu}$ halbieren. ${ }^{30}$

Ein anderer spezieller Themenbereich, in dem aktuell Beratungsbedarf seitens Politik und Verwaltung gesehen wird und in dem viele der bestehenden Ernährungsräte in Deutschland bereits aktiv sind, ist die öffentliche Gemeinschaftsverpflegung mit einem besonderen Schwerpunkt auf Schulen und Kitas. In mehreren Städten werden Ernährungsräte bereits in städtische Arbeitsgruppen miteinbezogen. Die Stadt Oldenburg entwickelt z. B. derzeit im Rahmen der Arbeitsgruppe „Mensakonzept" Kriterien zur Vergabe von Aufträgen an Caterer für die Schulverpflegung. In dieser Arbeitsgruppe ist der Ernährungsrat mit einer Person vertreten, die sich dafür einsetzt, dass soziale und ökologische Kriterien bei der Konzeptentwicklung berücksichtigt werden. ${ }^{31}$ Der Ernährungsrat Oldenburg hat in diesem Zusammenhang ein Positionspapier mit Vorschlägen und Forderungen erarbeitet. ${ }^{32}$ Diese Forderungen bilden die aus Sicht des Ernährungsrats relevanten Aspekte ab und bieten konkrete Ansatzpunkte, wie die öffentliche Gemeinschaftsverpflegung der Stadt im Hinblick auf Nachhaltigkeit verbessert werden könnte.

Die Ausführungen verdeutlichen, dass Ernährungsräte bisher nicht kontinuierlich von Politik und Verwaltung zur Beratung herangezogen werden, aber punktuell und themenspezifisch, wie derzeit von mehreren Städten bei der Verbesserung von Gemeinschaftsverpflegung. Die von mehreren Bundesländern initiierten Runden Tische zum Thema Lebensmittelwertschätzung weisen auf ein zunehmendes Interesse an der Beratung durch verschiedene gesellschaftliche Akteure im Ernährungsbereich hin.

\section{Rechtliche Verankerung von Ernährungsräten in Deutschland}

\subsection{Derzeitiger rechtlicher Status von Ernährungsräten}

Die bisher in Deutschland gegründeten Ernährungsräte sind mit wenigen Ausnahmen (s. Kapitel 3.1) aus zivilgesellschaftlicher Initiative entstanden. Teilweise sind die Räte zivilrechtlich organisiert als eigenständiger Verein (z. B. in Berlin und München) oder als Projekt eines vorher bereits bestehenden Vereins (z. B. in Köln und in Oldenburg). In anderen Fällen sind die Räte als lose zivilgesellschaftliche Bündnisse organisiert. Auch wenn in vielen Fällen Vertreterinnen und Vertreter aus Politik und Verwaltung schon in der Gründungsphase involviert waren, sind die Ernährungsräte bisher im öffentlich-rechtlichen Sinne formal nicht relevant.

Rein zivilgesellschaftlich organisiert zu sein ist durchaus typisch für das institutionelle Phänomen der Ernäh- rungsräte: Nach einer aktuellen Erhebung zum Status der Ernährungsräte in den USA und Kanada sind 34 Prozent eingebettet in eine andere zivilgesellschaftliche Organisation, 20 Prozent agieren als unabhängiger Graswurzelzusammenschluss und 13 Prozent als eigenständige zivilgesellschaftliche Organisation. Dennoch sind auch 26 Prozent der dortigen Ernährungsräte staatlich eingebettet und somit Teil des politisch-administrativen Gefüges. ${ }^{33}$ Von diesen staatlich eingebetteten Ernährungsräten ist ungefähr die Hälfte auf der Grundlage eines Gesetzes entstanden. Mehrheitlich werden die Mitglieder dieser Räte von staatlicher Seite benannt und die Gremien werden außerdem in den meisten Fällen von staatlicher Seite finanziell unterstützt. ${ }^{34}$

\subsection{Möglicher rechtlicher Status von Ernährungsräten de lege lata und de lege ferenda}

Die bisher in Deutschland gegründeten Ernährungsräte agieren wie dargelegt ohne öffentlich-rechtliche Grundlage. Dennoch stellt sich die Frage, ob unter den derzeitigen rechtlichen Rahmenbedingungen bereits die Möglichkeit bestünde, Ernährungsräte auch in Deutschland staatlich/ kommunal einzubetten bzw. unter welchen Umständen eine rechtliche Einbettung zukünftig denkbar wäre. Die Ergebnisse der Analyse sind unten in Tabelle 1 zusammengefasst.

Da es sich bei Ernährungsräten um Gremien handelt, die langfristig Politik beraten, können sie nicht mit Kommissionen gleichgesetzt werden, die für einen begrenzten zeitlichen Rahmen eingesetzt werden, um in Hinblick auf spezifische Beratungsanliegen zu beraten, wie z.B. die Rürup-Kommission. Weder auf Bundes- noch auf Länderebene gibt es gesetzliche Grundlagen, aufgrund derer Beratungsgremien, die nicht ausschließlich aus gewählten Vertreterinnen und

29) Bundesministerium für Ernährung und Landwirtschaft, Nationale Strategie zur Reduzierung der Lebensmittelverschwendung 2019, Stand 4.7.2019, abrufbar unter https://www.bmel.de/ SharedDocs/Downloads/Ernaehrung/Nationale_Strategie_Lebensmittelverschwendung_2019.html, S. 9.

30) S. Bundesministerium für Ernährung und Landwirtschaft, $\mathrm{Na}$ tionale Strategie zur Reduzierung der Lebensmittelverschwendung 2019, Stand 4.7.2019, abrufbar unter https://www. bmel.de/SharedDocs/Downloads/Ernaehrung/Nationale_Strategie_Lebensmittelverschwendung_2019.html, S. 5.

31) Ernährungsrat Oldenburg, Projekt Außer-Haus-Verpflegung, Stand 4.7.2019, abrufbar unter https://ernaehrungsrat-oldenburg.de/ueber-uns/projekte/ausser-haus-verpflegung/.

32) Ernährungsrat Oldenburg, 2019, Positionspapier Städtische Gemeinschaftsverpflegung als Vorbild für ein zukunftsfähiges Ernährungssystem, Stand 4.7.2019, abrufbar unter https://ernaehrungsrat-oldenburg.de/wp-content/uploads/2019/05/Positionspapier-St\%C3\%A4dtische-Gemeinschaftsverpflegung.pdf.

33) Bassarab/Santo/Palmer, 2019, Food Policy Council Report 2018 , Stand 1.7.2019, abrufbar unter http://www.foodpolicynetworks.org/food-policy-resources/, S. 11.

34) Ebenda, S. 16 
Vertretern bestehen, längerfristig eingesetzt werden. Auf der kommunalen Ebene ist hingegen die Einrichtung von verschiedenen beratenden Beiräten und Ausschüssen durchaus vorgesehen. Prinzipiell wird zwischen Pflichtausschüssen, bedingten Pflichtausschüssen und freiwilligen Ausschüssen unterschieden. Pflichtausschüsse müssen von den Gemeinden eingerichtet werden, bedingte Pflichtausschüsse nur in dem Fall, dass sich die Gemeinde einer bestimmten Angelegenheit annimmt. Wenn es keine gesetzlichen Vorgaben gibt, kann die jeweilige Volksvertretung selbst über das $\mathrm{Ob}$ und die Anzahl von Ausschüssen entscheiden. ${ }^{35}$ In den Gemeindeordnungen wird zwischen beschließenden und beratenden Ausschüssen unterschieden. In allen Bundesländern mit Ausnahme Sachsen-Anhalts können sowohl beschließende Ausschüsse als auch beratende Ausschüsse durch schlichten Gemeindebeschluss gebildet werden. ${ }^{36} \mathrm{Be}-$ schließende Ausschüsse können nur aus gewählten Vertreterinnen und Vertretern bestehen, da sie demokratisch legitimiert sind. Die Vertretung kann aber beratende Ausschüsse einrichten, in denen neben den gewählten Vertreterinnen und Vertretern auch nicht-gewählte Mitglieder angehören. In $\$ 71$ Abs. $7 \mathrm{NKomVG}^{37}$ ist z. B. geregelt, dass mindestens zwei Drittel der Mitglieder dieser beratenden Ausschüsse Abgeordnete sein sollen, darüber hinaus aber Mitglieder im Ausschuss sein können, die nicht der Vertretung angehören. Letztere haben allerdings auch kein Stimmrecht. Nach $₫ 43$ Abs. 4 BbgKVerf ${ }^{38}$ können sog. sachkundige Einwohner zu beratenden Mitgliedern der Ausschüsse berufen werden und haben dann ein aktives Teilnahmerecht in dem Ausschuss. Auch nach $\$ 36$ Abs. 5 KV M-V ${ }^{39}$ können neben einer Mehrheit von Mitgliedern der Gemeindevertretung weitere sachkundige Einwohner in die beratenden Ausschüsse berufen werden. Ähnliches gilt für verschiedene andere Kommunalverfassungen, so etwa nach $\$ 41$ Abs. $1 \mathrm{GO} B-W,{ }^{40} \$ 49$ Abs. 3 KVG LSA ${ }^{41}$ oder $\ 58$ Abs. 3 GO NRW..$^{42}$ Die sachkundigen Bürger kommen nach diesen Regelungen zu den gewählten Ratsmitgliedern im jeweiligen Ausschuss hinzu, der Ausschuss ist aber ,nur beschlussfähig, wenn die Zahl der anwesenden Ratsmitglieder die Zahl der anwesenden sachkundigen Bürger übersteigt" (so $\$ 58$ Abs. 3 GO NRW).

Nach diesen kommunalverfassungsrechtlichen Vorgaben können z. B. Ernährungsräte als Ausschüsse der Städteund Gemeinderäte errichtet werden, die in ihrer Mehrzahl aus gewählten Mitgliedern bestehen, zu denen aber weitere nicht dem Rat angehörige, in Ernährungsfragen sachkundige Bürger hinzukommen können. Die Bestellung erfolgt regelmäßig durch den Rat mit der Mehrheit der Stimmen der Ratsmitglieder (so nach $₫ 58$ Abs. 1 GO NRW). Mit der Bestellung können die Aufgaben eines kommunalen Ernährungsrats bestimmt werden, z. B. welche Stufen in der Wertschöpfungskette abgedeckt werden sollen. Notwendiger Inhalt der Bestellung wären zudem $\mathrm{Zahl}$ und $\mathrm{Zu}-$ sammensetzung des Ernährungsrats. Weiter kann festgelegt werden, in welcher Weise der kommunale Ernährungsrat an Entscheidungen des Rates beteiligt wird, ob er z. B. bei bestimmten ernährungsbezogenen Angelegenheiten angehört werden muss.

Darüber hinaus existieren auf kommunaler Ebene in der Regel Beiräte, die den Stadt- oder Gemeinderat beraten, in denen aber keine Ratsmitglieder vertreten sind, wie z.B. Ausländerbeiräte oder Jugendvertretungen. ${ }^{43}$ Diese Beiräte werden auf der Grundlage eines Gesetzes bzw. einer Verordnung, in der die Anzahl und Zusammensetzung der Mitglieder geregelt ist, eingerichtet.

Unter den bestehenden gesetzlichen Rahmenbedingungen ist die Einrichtung von langfristigen beratenden Gremien, die auch bzw. nur nicht gewählte Vertreterinnen und Vertreter umfassen, nur auf der kommunalen Ebene vorgesehen. Die Einrichtung beratender Beiräte auf Bundes- oder Landesebene beruht zurzeit nicht auf gesetzlicher Grundlage, sondern auf der Organisationsgewalt der einsetzenden Stellen. Ein Beispiel für ein Beratungsgremium auf Bun- desebene ist der Parlamentarische Beirat für nachhaltige Entwicklung (BPnE), der 2004 beschlossen und auch in der laufenden Legislaturperiode erneut eingesetzt wurde, um , [...] die Nachhaltigkeitspolitik der Bundesregierung auf parlamentarischer Ebene in geeigneter Weise fachübergreifend zu begleiten". ${ }^{44}$ Dieser besteht ausschließlich aus Mitgliedern der Fraktionen entsprechend der Sitzverteilung im Parlament. ${ }^{45}$ Aus nicht-gewählten Personen setzt sich der Rat für nachhaltige Entwicklung (RNE) zusammen, der 2001 erstmalig von der Bundesregierung berufen wurde. Zuletzt wurden von der Bundeskanzlerin im Jahr 201615 Personen des öffentlichen Lebens als Mitglieder für eine dreijährige Amtsperiode berufen. Darüber hinaus kann der RNE für die Dauer von Projekten Persönlichkeiten mit spezieller Fachexpertise kooptieren. ${ }^{46}$ Der RNE hat die Aufgabe, Beiträge für die Umsetzung der Deutschen Nachhaltigkeitsstrategie zu entwickeln, konkrete Handlungsfelder und Projekte zu benennen sowie Nachhaltigkeit zu einem wichtigen öffentlichen Anliegen zu machen. Dabei ist er in der Wahl seiner Themen und Aktionsformen unabhängig und verfügt zur Durchführung seiner Aufgaben über eigene finanzielle Mittel. ${ }^{47}$

Mit dem wissenschaftlichen Beirat für Agrarpolitik, Ernährung und gesundheitlichen Verbraucherschutz (WBAE) gibt es seit 2015 auf Bundesebene auch ein unter anderem auf die Ernährungspolitik fokussiertes, ausschließlich professoral mit 19 Mitgliedern besetztes Gremium, dessen derzeitige Mitglieder durch das Bundesministerium für Ernährung und Landwirtschaft bestellt wurden. Zu dessen Aufgaben gehört es unter anderem, ,,die Entwicklung des Agrar- und Ernährungssystems in Bezug auf seine wirtschaftliche, ökologische und soziale Nachhaltigkeit wissenschaftlich zu analysieren", zu bewerten sowie „Vorschläge für die Weiterentwicklung der Agrar- und Ernährungspolitik zu erarbeiten". ${ }^{48}$ Der WBAE hat sich in diversen Veröffentlichungen auch mit Fragen der Ernährungspolitik befasst, z.B. 2012 in einer Stellungnahme zu „Ernährungs-

35) Ehlers, in: Mann/Püttner (Hrsg.), Handbuch der kommunalen Wissenschaft und Praxis, 2007, S. $495 \mathrm{f}$.

36) Wolff/Bachof/Stober/Kluth, Verwaltungsrecht 2, 7. Aufl. 2010, S. 741.

37) Niedersächsisches Kommunalverfassungsgesetz (NKomVG) v. 17. 10.2010, Nds. GVBl. S. 576.

38) Kommunalverfassung des Landes Brandenburg (BbgKVerf) v. 18.12.2007, GVBl. I/07, [Nr. 19], S. 286.

39) Kommunalverfassung für das Land Mecklenburg-Vorpommern (Kommunalverfassung - KV M-V) v. 13.7.2011, GVOBl. M-V S. 777.

40) Gemeindeordnung In der Fassung vom 24.7.2000, GB1. S. 582.

41) Kommunalverfassungsgesetz des Landes Sachsen-Anhalt (Kommunalverfassungsgesetz - KVG LSA) v. 17.6.2014, GVBl. LSA S. 288.

42) Gemeindeordnung für das Land Nordrhein-Westfalen (GO NRW) in der Fassung der Bekanntmachung v. 14.7.1994, GV. NRW. S. 666.

43) S. Ehlers, in: Mann/Püttner (Hrsg.), Handbuch der kommunalen Wissenschaft und Praxis, 2007, S. $495 \mathrm{f}$

44) S. den Antrag der Fraktionen CDU/CSU, SPD, AfD, FDP, DIE LINKE und BÜNDNIS 90/DIE GRÜNEN auf Einsetzung des Parlamentarischen Beirats für nachhaltige Entwicklung, BTDrs. 19/1837 vom 24. 4.2018, S. 1.

45) S.S. den Antrag der Fraktionen CDU/CSU, SPD, AfD, FDP, DIE LINKE und BÜNDNIS 90/DIE GRÜNEN auf Einsetzung des Parlamentarischen Beirats für nachhaltige Entwicklung, BTDrs. 19/1837 vom 24. 4. 2018, S. 1.

46) Rat für nachhaltige Entwicklung (RNE), Mitglieder, Stand 3.7.2019, abrufbar unter https://www.nachhaltigkeitsrat.de/ueber-den-rat/mitglieder/.

47) Rat für nachhaltige Entwicklung (RNE), Über den Rat, Stand 3.7.2019, abrufbar unter https://www.nachhaltigkeitsrat.de/ueber-den-rat/.

48) Bundesministerium für Ernährung und Landwirtschaft, Wissenschaftlicher Beirat für Agrarpolitik, Ernährung und gesundheitlichen Verbraucherschutz, Stand 16.8.2020, abrufbar unter https://www.bmel.de/DE/Ministerium/Organisation/Beiraete/_Texte/AgrOrganisation.html\#doc429078bodyText1. 
sicherung und nachhaltige Produktivitätssteigerung". ${ }^{49}$ Mit seiner ausschließlich auf Beratung durch die Wissenschaft gestützten Ausrichtung entspricht der WBAE nicht dem Muster eines Ernährungsrats im hier vertretenen Sinne.

Auf Landesebene sind die Naturschutzbeiräte ein Beispiel für beratende Gremien mit pluralistischer Besetzung. Diese Beiräte werden auf Grundlage der Landesnaturschutzgesetze auf verschiedenen Ebenen der Naturschutzverwaltung eingesetzt. In den Landesbeirat für Natur- und Umweltschutz in Baden-Württemberg werden gemäß einer Verordnung des Umweltministeriums z.B. neben je einem Mitglied aus den Fraktionen Personen aus verschiedenen gesellschaftlichen Bereichen wie der Naturschutzvereinigungen, Verbraucherverbände, Kirchen oder des Sports berufen, um das für zuständige Ministerium in Fragen des Naturschutzes zu beraten. ${ }^{50}$ Das Vorschlagsrecht für die verschiedenen Bereiche liegt nach $\$ 4$ der Verordnung bei den zuständigen Verbänden.

Die vorgestellten Beispiele verdeutlichen, dass bei entsprechenden politischen Mehrheiten auch auf Bundesund Landesebene Beiräte eingerichtet werden könnten, die pluralistisch besetzt sind und die Politik und Verwaltung in Bezug auf Ernährungsfragen beraten könnten. Die Einrichtung solcher Beiräte durch oder aufgrund eines Gesetzes hat den Vorteil einer besseren Verankerung und größeren Unabhängigkeit des Beirats. Dagegen hat die Einsetzung durch einen Organisationsakt der jeweiligen Regierung den Nachteil einer größeren Abhängigkeit. Auf der anderen Seite ist dies aber weniger aufwendig, da es keines Gesetzgebungsverfahrens, sondern eines einfachen Regierungs- bzw. Ministeriumsbeschlusses bedürfte.

\section{Pro und Contra einer stärkeren rechtlichen Verankerung von Ernährungsräten}

Die Analyse der rechtlichen Rahmenbedingungen hat verdeutlicht, dass auf kommunaler Ebene bereits jetzt beratende Beiräte eingerichtet werden können, die nicht bzw. nicht ausschließlich aus gewählten Vertreterinnen und Vertretern bestehen. Auf Bundes- und Landesebene könnten auf Gesetzesinitiative hin oder durch einfachen Regierungsbeschluss ebenfalls entsprechende beratende Gremien eingerichtet werden. Es stellt sich allerdings die Frage, ob eine stärkere rechtliche Einbettung der bereits gegründeten Ernährungsräte überhaupt sinnvoll ist, sowohl aus Sicht derer, die beraten als auch aus Sicht derer, die beraten werden. Grundvoraussetzung wäre seitens der Politik eine Mehrheit dafür, in Ernährung im umfassenden Sinn, d.h. in Bezug auf Produktion, Verarbeitung, Vertrieb, Konsum und Verwertung sowie die in der Einleitung dargestellten Bezüge zu Umwelt, Gesundheit, Verbraucherschutz und Regionalentwicklung überhaupt ein Beratungsanliegen zu sehen und Ernährungsräte als Beratungsinstrument anzuerkennen.

Die in Kapitel 3 vorgestellten Beispiele haben auch verdeutlicht, dass Ernährungsräte im speziellen Themenfeld der Gemeinschaftsverpflegung bereits von Politik und Verwaltung zur Beratung herangezogen werden. Auch im Bereich Lebensmittelwertschätzung wurde von mehreren Ländern mit der Einrichtung von Runden Tischen bereits ein Instrument benutzt, um möglichst viele Personen aus dem Ernährungsbereich punktuell zu involvieren. Die Verbesserung der Gemeinschaftsverpflegung und die Reduzierung von Lebensmittelabfällen sind allerdings nur zwei von vielen Themen, mit denen sich Ernährungsräte beschäftigen. Die Nutzung bzw. Ausweisung städtischer Flächen für Nutzpflanzen oder gemeinschaftliche Gartenprojekte, die Stärkung regionaler Erzeugerinnen und Erzeuger, die Förderung von Ernährungskompetenzen in der Bevölkerung sind nur einige Beispiele, die das breite thematische Spektrum von Ernährungsräten verdeutlichen. Mit ihren vielfältigen Aktivitäten geben Ernährungsräte zahlreiche Ansatzpunkte für die Gestaltung einer Ernährungswende und tangieren weitere Handlungsbereiche, in denen die ver- schiedenen politisch-administrativen Ebenen Handlungsspielraum haben wie z. B. die Stadtplanung in Hinblick auf Flächennutzung oder Initiative ergreifen können, wie z. B. bei der Förderung regionaler Vermarktungsstrukturen.

Vor diesem Hintergrund könnte eine stärkere rechtliche Einbettung von Ernährungsräten dazu beitragen, dass die Belange einer Ernährungswende politisch vermehrt angegangen werden und in der Öffentlichkeit über den Bereich der ohnehin Engagierten bekannter würden. Wie in $\mathrm{Ka}$ pitel 4 dargelegt, wäre es bei entsprechenden politischen Mehrheiten grundsätzlich möglich, Ernährungsräte als beratende Beiräte auf verschiedenen politisch-administrativen Ebenen einzurichten. Dies würde z.B. beinhalten, dass die Zusammensetzung der jeweiligen Gremien geregelt würde sowie die Art und Weise, in der sie beraten (z. B. Anhörung in thematisch relevanten Ausschüssen) und die Gremien ggf. eine finanzielle Aufwandsentschädigung bekämen, um ihrem Mandat nachzukommen.

Eine derartige Institutionalisierung würde andererseits aber die Offenheit der bestehenden Ernährungsräte und Gründungsinitiativen einschränken und liefe damit Gefahr, die dynamische Entwicklung von Ernährungsräten aus der Zivilgesellschaft heraus zu bremsen. In jedem Fall sollte eine stärkere rechtliche Einbettung bestehender Ernährungsräte nur in Abstimmung mit den bereits Beteiligten geschehen. Wie oben dargelegt variieren die Selbstverständnisse der Initiativen, und teilweise gibt es auch innerhalb der Initiativen verschiedene Auffassungen, auf welche Weise eine $\mathrm{Zu}-$ sammenarbeit mit Politik und Verwaltung sinnvoll ist.

Vor diesem Hintergrund erscheint es adäquat, in jedem Einzelfall genau auszuloten, wie seitens Politik und Verwaltung gesellschaftliches Engagement für eine Ernährungswende gefördert und ggf. auch initiiert werden kann. Besteht noch keine Ernährungsratsinitiative, könnten Fraktionen wie im Fall der Stadt Bielefeld die Gründung anregen und die Verwaltung beauftragen, diesen Prozess zu begleiten und organisatorisch zu unterstützen. Bereits bestehende Gründungsinitiativen könnten durch Fördergelder für Koordinationspersonen, den Aufbau von Infrastrukturen und Veranstaltungen unterstützt werden, wie zum Beispiel beim ersten Ernährungsrats Niedersachsens in Oldenburg durch Landesmittel. ${ }^{51}$ Bereits gegründete Ernährungsräte könnten z.B. durch die Einladung, ihre Anliegen in Ausschüssen vorzutragen oder in städtischen Arbeitsgruppen mitzuarbeiten, wie es im Bereich Gemeinschaftsverpflegung bereits in mehreren Städten praktiziert wird, unterstützt werden, sowie durch eine längerfristige institutionelle Förderung wie im Fall der Stadt Köln, wo der Stadtrat 2017 beschlossen hat, die Arbeit des dortigen Ernährungsrats für weitere drei Jahre finanziell zu unterstützen. ${ }^{52}$

\section{Fazit}

Ernährungsräte greifen ein Thema auf, das viele Menschen bewegt und in den Debatten um Klimawandel zu-

49) WBAE,Stellungnahmezu,,Ernährungssicherungundnachhaltige Produktivitätssteigerung",Abrufbarunterhttps://www.bmel.de/ SharedDocs/Downloads/Ministerium/Beiraete/Agrarpolitik/ Stellungnahme-Ern\%C3\%A4hrungssicherung.html.

50) $\$ 1$ und $\$ 2$ der Verordnung des Umweltministeriums über den Landesbeirat für Natur- und Umweltschutz (BeiratsVO Natur und Umwelt) v. 5. 4.2017, GB1. S. 241.

51) Weitere Aspekte zum Gründungsprozess des Ernährungsrats Oldenburg, der im Rahmen der Doktorarbeit von Annelie Sieveking in einer zweijährigen Fallstudie untersucht wurde, in Sieveking, Food Policy Councils as Loci for Practising Food Democracy? Insights from the Case of Oldenburg, Germany, Politics and Governance 7 (4), 2019, S. 48-58.

52) Amt für Presse- und Öffentlichkeitsarbeit der Stadt Köln, Presseinformation 12.7.2017 - 1032 Zuschuss für Ernährungsrat Köln und Umgebung. 
nehmend an Bedeutung gewinnt. Sie sind eine beginnende zivilgesellschaftliche Bewegung, die bisher nicht im öffentlich-rechtlichen Sinne verankert sind. Ihr derzeitiger Schwerpunkt liegt auf der städtischen/kommunalen Ebene, während die Länder- und Bundesebene, vor allem auch wegen des Graswurzel-Charakters vieler Ernährungsräte, bisher kaum eine Rolle spielen. Allerdings verbreitet sich derzeit die Idee, Ernährungsräte auch auf höheren politisch-administrativen Ebenen einzusetzen. Im kürzlich veröffentlichten Bericht des International Panel of Experts on Sustainable Food Systems (iPES Food) wird die Unterstützung der Etablierung eines Ernährungsrats auf EU-Ebene als eine politische Maßnahme im Rahmen einer neuen Governance-Architektur für nachhaltige Ernährungssysteme vorgeschlagen. ${ }^{53}$

Möglicherweise kann eine stärkere rechtliche Einbindung von Ernährungsräten zu einer besseren Durchsetzung der Belange im Hinblick auf eine Ernährungswende führen und z.B. zur Verringerung von Lebensmittelabfällen beitragen. Auf der anderen Seite sollte eine solche Bewegung von unten nicht durch zu viele formale Anforderungen abgewürgt werden. Wünschenswert wäre, wenn öffentliche Entscheidungsträger zumindest Bereitschaft zeigen würden, die Belange von Ernährungsräten anzuhören und das zivilgesellschaftliche Engagement im Rahmen ihrer Möglichkeiten zu unterstützen. Dies gilt z.B. für die Förderung von Projekten, ggf. durch Abordnung von Mitarbeitern aus der Verwaltung zur Mitarbeit in den Räten, für die Bereitstellung von Räumlichkeiten und Koordinationsstellen etc. Ein wichtiges Signal kann weiter darin bestehen, wenn Kommunen dem Mailänder Abkommen über städtische Ernährungspolitik (Milan Urban Food Policy Pact) vom 15.10.2015 als politischem Bezugspunkt für proaktives Handeln beitreten. Hierin anerkennen die Mitgliedsstädte unter anderem deren zentrale Rolle bei der Entwicklung nachhaltiger Ernährungssysteme, und sie erklären, dass sie ihre kommunale Ernährungspolitik in ihre sozialen, ökonomischen und Umweltpolitiken sowie -programme integrieren. ${ }^{54}$ Bisher haben 197 Städte den Pakt unterzeichnet, darunter allerdings nur drei deutsche: Frankfurt, Köln und Berlin (2015). Ernährungsräte können dazu beitragen, ihre jeweiligen Kommunen zur Teilnahme an diesem Pakt zu bewegen und im Hinblick auf eine Ernährungswende aktiv zu werden.

Insgesamt bieten Ernährungsräte die Chance, in der gesamten Kette, von der Erzeugung über die Verarbeitung und den Transport bis zum Verbrauch und zur Vermeidung und Entsorgung von Lebensmittelabfällen, eine Wende in Richtung auf eine nachhaltigere Ernährung zu fördern. Dazu bedarf es zivilgesellschaftlichen Engagements und der Aufgeschlossenheit und Bereitschaft privater und öffentlicher Stellen, die Bildung und Arbeit von Ernährungsräten zu unterstützen - nicht zuletzt auch durch die Schaffung geeigneter rechtlicher Rahmenbedingungen.

Open Access. Dieser Artikel wird unter der Creative Commons Namensnennung 4.0 International Lizenz veröffentlicht, welche die Nutzung, Vervielfältigung, Bearbeitung, Verbreitung und Wiedergabe in jeglichem Medium und Format erlaubt, sofern Sie den/die ursprünglichen Autor(en) und die Quelle ordnungsgemäß nennen, einen Link zur Creative Commons Lizenz beifügen und angeben, ob Änderungen vorgenommen wurden.

Die in diesem Artikel enthaltenen Bilder und sonstiges Drittmaterial unterliegen ebenfalls der genannten Creative Commons Lizenz, sofern sich aus der Abbildungslegende nichts anderes ergibt. Sofern das betreffende Material nicht unter der genannten Creative Commons Lizenz steht und die betreffende Handlung nicht nach gesetzlichen Vorschriften erlaubt ist, ist für die oben aufgeführten Weiterverwendungen des Materials die Einwilligung des jeweiligen Rechteinhabers einzuholen.

Weitere Details zur Lizenz entnehmen Sie bitte der Lizenzinformation auf http://creativecommons.org/licenses/by/4.0/deed.de.

Open Access funding enabled and organized by Projekt DEAL.

53) De Schutter/Jacobs/Clément/Ajena, Towards a Common Food Policy for the European Union, 2019, Stand 16.8.2020, abrufbar unter http://www.ipes-food.org/pages/CommonFoodPolicy, S. 36-37.

54) Milan Urban Food Policy Pact, Stand 5.7.2019, abrufbar unter http://www.milanurbanfoodpolicypact.org/signatory-cities/.

\title{
Europäisierung des Individualrechtsschutzes im Umweltrecht
}

\author{
Anmerkung zum Urteil des Europäischen Gerichtshofs v. 3.10.2019 - C-197/18 \\ Dominik Römling
}

(c) Der/die Autor(en) 2020. Dieser Artikel ist eine Open-Access-Publikation.

Während die Fortentwicklung des Verbandsklagerechts nach Art. 9 Abs. 3 der Aarhus-Konvention mit der Protect-Entscheidung des Europäischen Gerichtshofs ihren vorläufigen Schlusspunkt erreicht hat, besteht die Diskussion um die Klagerechte Einzelner aus der

Dominik Römling, Wissenschaftlicher Mitarbeiter

am Institut für Umwelt- und Planungsrecht,

Westfälische Wilhelms-Universität,

Münster, Deutschland
Konvention fort. Sie erfährt jüngst durch das Urteil des EuGH in der Rs. C-197/18 neue Impulse. Der Beitrag analysiert die Reichweite der dort entwickelten Maßstäbe und legt die Konsequenzen für das Verständnis des Individualrechtsschutzes im deutschen Umweltrecht dar.

\section{Einführung}

Art. 9 Abs. 3 der Aarhus-Konvention (AK) hat die Klagerechte von Verbänden in erheblichem Maße erwei- 